【Transaction】

\title{
Preparation of Anti-haze Composite Window Screen Based on Nanofibers and Study on the Composite Process Parameters
}

\author{
Weili Shao*1, Wanjun Bu*1, Tong Lu*1, Junli Li*1, Hewei Xiang*1,

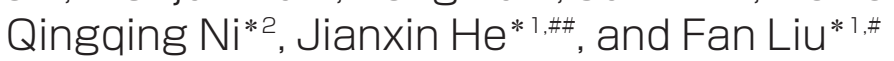 \\ ${ }^{* 1}$ Textile and Clothing Industry Research Institute, Zhongyuan University of Technology, Zhengzhou 450007, Henan; \\ ${ }^{* 2}$ Department of Functional Machinery \& Mechanics, Shinshu University, Ueda 310018, Japan
}

\begin{abstract}
As air pollution has become increasingly serious, anti-haze filter materials have received considerable attention. In this study, a composite window screen with a sandwich structure consisting of a polyacrylonitrile (PAN) nanofiber membrane as the filter layer, a 30 mesh glass fiber mesh as the supporting layer, and a 120 mesh polyester fiber mesh as the protective layer was prepared. We analyzed the effects of different composite methods and composite parameters on the performance of the anti-haze window screen. The results showed that with an increase in the ultrasonic pressure, the peel strength increased, but the nanofiber membrane was damaged by the ultrasonic treatment and the filterability decreased. Using a hot melt adhesive compounding process, the peel strength of the composite window screen increased with an increase in adhesive amount; however, the filtration efficiency remained nearly constant at $95 \%$, despite the increase in the filtration resistance. The optimal amount of adhesive was confirmed as $1.2 \mathrm{~g} / \mathrm{m}^{2}$. In addition, the hot melt adhesive composite technology is simple and controllable, which is beneficial for the industrial production of anti-haze window screens.
\end{abstract}

(Received 4 June, 2021; Accepted 17 July, 2021)

\section{Introduction}

With the rapid development of global industrialization, haze weather conditions are becoming increasingly serious worldwide, which has attracted significant attention from the public. According to a series of reports released by the UN Environment Programme, haze causes seven million deaths every year worldwide; among them, 4.3 million people die of indoor air pollution [1]. Among air pollutants, particulate matter (PM), especially $\mathrm{PM}_{2.5}$ (particles with a diameter less than or equal to $2.5 \mu \mathrm{m}$ ), is the most harmful to humans. These particles can combine with other substances in the air and remain airborne for extended periods. They can be easily inhaled and may cause allergies or respiratory infectious diseases [2-4]. Considering the long-term indoor activities of people, reducing indoor $\mathrm{PM}_{2.5}$ is an effective method to protect human health. At present, the most widely used air purification method is to remove $\mathrm{PM}_{2.5}$ particles using air-cleaners. However, air-cleaners only circulate and filter indoor air and cannot supply fresh air; thus, opening windows for ventilation is still required [5, 6]. Mashes mode of glass fiber, metal, etc. are commonly used window screen materials. However, the mesh of these window screens is on the millimeter scale, which can block mosquitoes and other small insects but cannot block $\mathrm{PM}_{2.5}$ [7]. The application of an ultra-fine fiber film as a window screen enables the appropriate filtering of $\mathrm{PM}_{2.5}$ while maintaining sufficient air permeability and light transmittance [8, 9].

Electrospinning is the most common and effective method for preparing nanofibers. Electrospun nanofiber membranes have the advantages of small pore size, high porosity, and good channel connectivity $[10,11]$. This enables the reduction of air permeability of materials; thus, it is considered to have great application potential in air filtration $[12,13]$. However, the strength of pure

\# corresponding author: Liu Fan (E-mail: liufan_zzti@126.com)

\#\# co-corresponding author: Jianxin He (E-mail: hejianxin771117@163.com) 


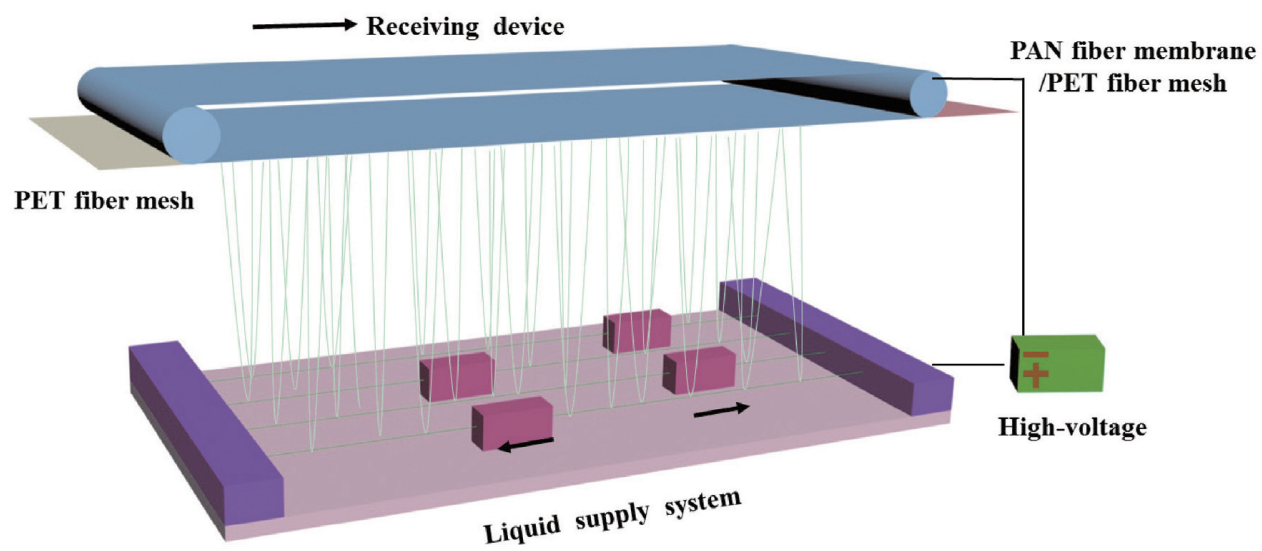

Fig. 1 Schematic diagram of the electrospinning setup

nanofiber membranes cannot meet the realistic requirements; thus, nanofiber membranes need to be combined with other substrate materials using composite technology.

In this study, a composite window screen with a sandwich structure was prepared, which consisted of a polyacrylonitrile (PAN) nanofiber membrane as the filter layer, a 30 mesh glass fiber mesh as the supporting layer, and a 120 mesh polyester fiber mesh as the protective layer. We studied the effect of different composite methods and composite parameters on the performance of anti-haze window screens and determined the optimal composite technology and parameters. This work aims to provide a solid foundation for the industrial production of nanofiber anti-haze window screens.

\section{Experimental Section}

\subsection{Materials}

Polyacrylonitrile $(\mathrm{Mr}=150,000)$ was provided by Tianjin Kemiou Chemical Reagent Co., Ltd., China, lithium chloride ( $\mathrm{LiCl}$ ) was provided by Tianjin Aopusheng Chemical Co., Ltd., China; N, Ndimethylformamide (DMF) was obtained from Xilong Scientific Co., Ltd., China, and polyurethane hot melt adhesive (PUR, particle size $75 \mu \mathrm{m}$ ) was supplied by the Shenzhen Renshan New Material Technology Co., Ltd., China. Both the 30 mesh glass fiber gauze and the 120 mesh polyester gauze were obtained from Shanghai Zhengxi New Material Technology Co., Ltd., China. All chemicals were of analytical grade and used without further purification.

\subsection{Electrospinning}

In the early stage of the study, we found that the optimal spinning concentration of PAN was $12 \%$ [14]. First, different $\mathrm{LiCl}$ amounts were dissolved in DMF and stirred for $12 \mathrm{~h}$ at $60{ }^{\circ} \mathrm{C}$. Then, quantitative PAN was added to the $\mathrm{LiCl} / \mathrm{DMF}$ solution, heated, and stirred for $24 \mathrm{~h}$ until the PAN was completely dissolved. Finally, a series of solutions with $\mathrm{LiCl}$ contents of 0 wt\%, 0.01 wt\%, 0.02 wt\%, and 0.03 wt\% were prepared.

Nanofiber membranes were prepared by linear electrode electrospinning using a 120 mesh polyester fiber mesh as the receiving base fabric, as shown in Fig. 1. The mesh cord speed was set to 150, 200, 250, 300 , and $350 \mathrm{~mm} / \mathrm{min}$ to obtain nanofiber membranes with different thicknesses. The spinning parameters were the same as those in our previous work: spinning voltage of $35 \mathrm{kV}$, spinning pitch of $18 \mathrm{~cm}$, temperature of $25{ }^{\circ} \mathrm{C}\left( \pm 3{ }^{\circ} \mathrm{C}\right)$, and humidity of $30 \%$

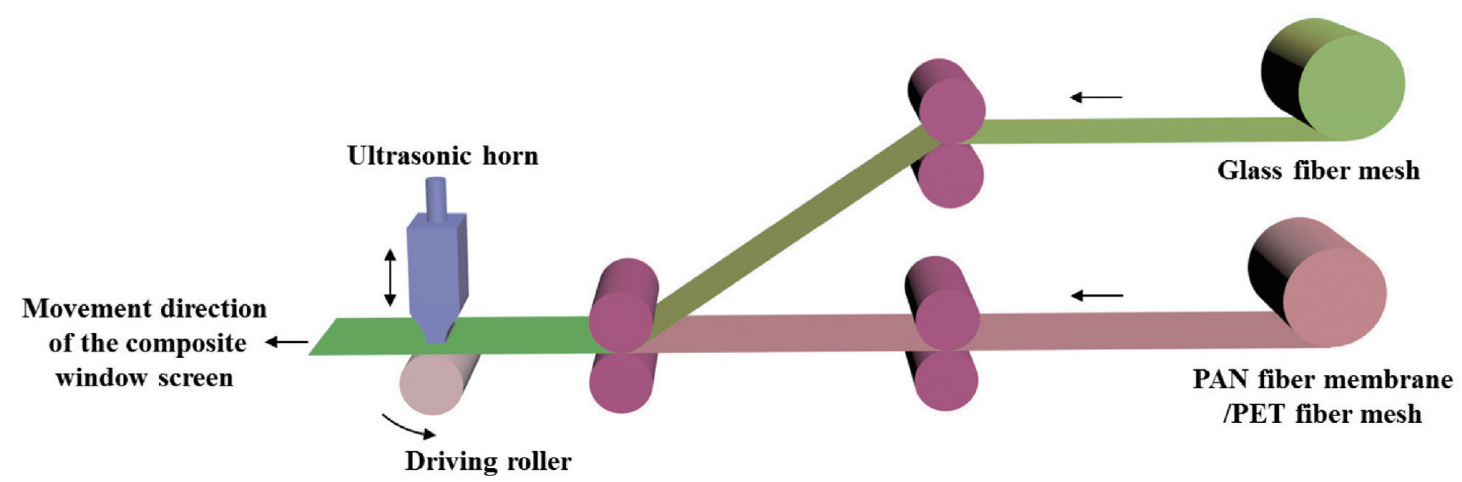

Fig. 2 Schematic diagram of the ultrasonic bonding machine 
$( \pm 5 \%)$.

\subsection{Ultrasonic composite process}

To composite the polyethylene terephthalate (PET) fiber mesh covered by nanofibers and the glass fiber mesh (Fig. 2), an ultrasonic bonding machine was used when the feeding speed of the compound reached the set value. The effects of different composite pressures $(0.5,1.5,2.6,3.9$, and $6 \mathrm{MPa})$ on the peel strength and filtration efficiency of the window screen were studied. The compounding time was $0.4 \mathrm{~s}$ and the holding pressure time was $0.6 \mathrm{~s}$.

\subsection{Hot melt adhesive composite process}

The PUR was coated on the surface of the glass fiber mesh using hot melt adhesive equipment and then bonded with the PET-fiber mesh with a nanofiber membrane, as shown in Fig. 3. Using this method, we prepared anti-haze window screens with different amounts of glue $\left(0.4,0.8,1.2,1.6\right.$, and $\left.2 \mathrm{~g} / \mathrm{m}^{2}\right)$, and studied the effects of the amount of glue on the peel strength and filtration performance. In addition, we studied the effect of PUR curing times $(4,8,12,24$, 36 , and $48 \mathrm{~h}$ ) on the peel strength. The recombination time and pressure were $13 \mathrm{~s}$ and $0.3 \mathrm{kPa}$, respectively, and the compound velocity was $5 \mathrm{~m} / \mathrm{min}$.

\subsection{Scanning electron microscopy test}

Scanning electron microscopy (SEM, Pw-100-515, Shanghai Funa Scientific Instruments Co., Ltd., China) was used to observe the morphology of the fiber membrane. The nanofiber membrane was applied to the sample table by a spraying current of $10 \mathrm{~mA}$ and scanning voltage of $10 \mathrm{kV}$. Fifty samples were randomly tested using a nanofiber measurer, and the average values were obtained.

\subsection{Filter performance test}

The filter performance of the nanofiber membrane was analyzed using an automatic filter material tester (8130A, TSI Incorporated, USA) at an air flow rate of $32 \mathrm{l} / \mathrm{min}$. The sample was cut into a circle with a diameter of $15 \mathrm{~cm}$, and the average value of ten tests for each sample was determined.

\subsection{Peel strength test}

A laminated ultra-thin fiber membrane automatic peel strength tester (QJ210A, Jinan Languang Electromechanical Technology Co., Ltd., China) was used to measure the peel strength, referring to the Chinese Standard GB/T 2790-1995. The specific peel strength test method was flexible versus rigid material. The sample was cut to a length of $260 \mathrm{~mm}$ and width of $50 \mathrm{~mm}$, the stretching speed was set to $500 \mathrm{~mm} / \mathrm{min}$, and the stretching distance was $250 \mathrm{~mm}$ [15]. In this experiment, the peel force at the maximum peak value was used as the value for calculating the peel strength, and the average value was obtained after five tests. The peel strength was calculated as

$$
\sigma 180^{\circ}=F / B,
$$

where $\sigma 180^{\circ}=180^{\circ}$ is the peel strength, $F$ is the maximum peak peel force, and $B$ is the width of the sample.

\section{Results and discussion}

The grid size of an ordinary window screen is approximately 1-20 mm (Fig. 4(a)), which can only block dust and mosquitoes; however, it provides no filtration for haze compounds with $\mathrm{PM}_{2.5}$. The core layer of a nanofiber composite window screen is a nanofiber non-woven membrane with pore sizes in the range of $0.5-2 \mu \mathrm{m}$ and with a three-dimensional structure (Fig. 4(b)), which has a good filtering effect for $\mathrm{PM}_{2.5}$.

\subsection{The effect of $\mathrm{LiCl}$ concentration on the diameter of PAN nanofibers}

The pore size of the nanofiber membrane is directly related to the fiber diameter, and the conductivity of the solution is one of the most

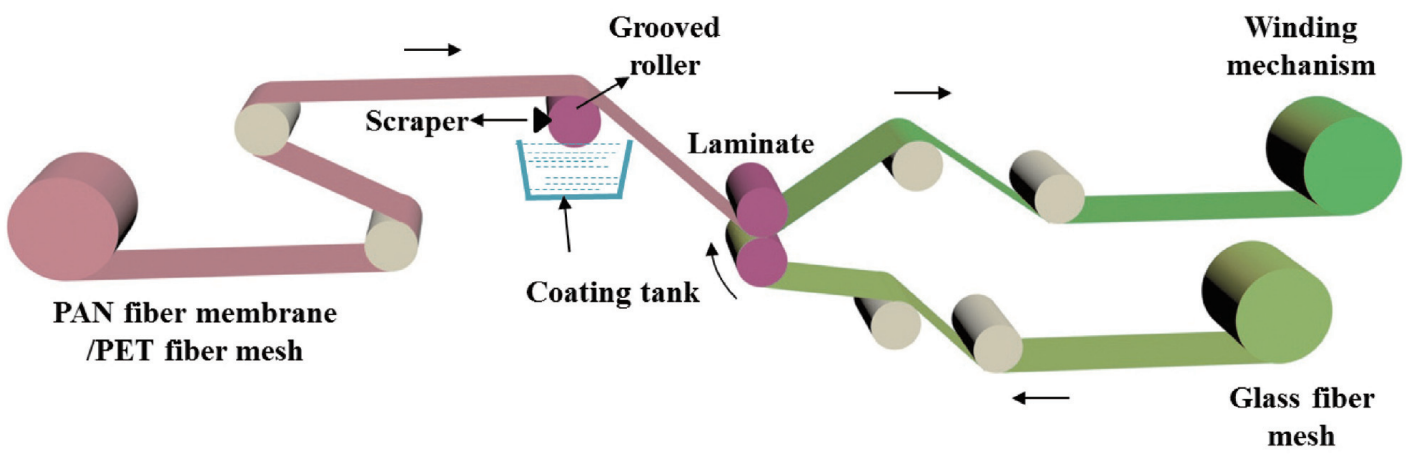

Fig. 3 Schematic diagram of hot melt adhesive laminating machine 

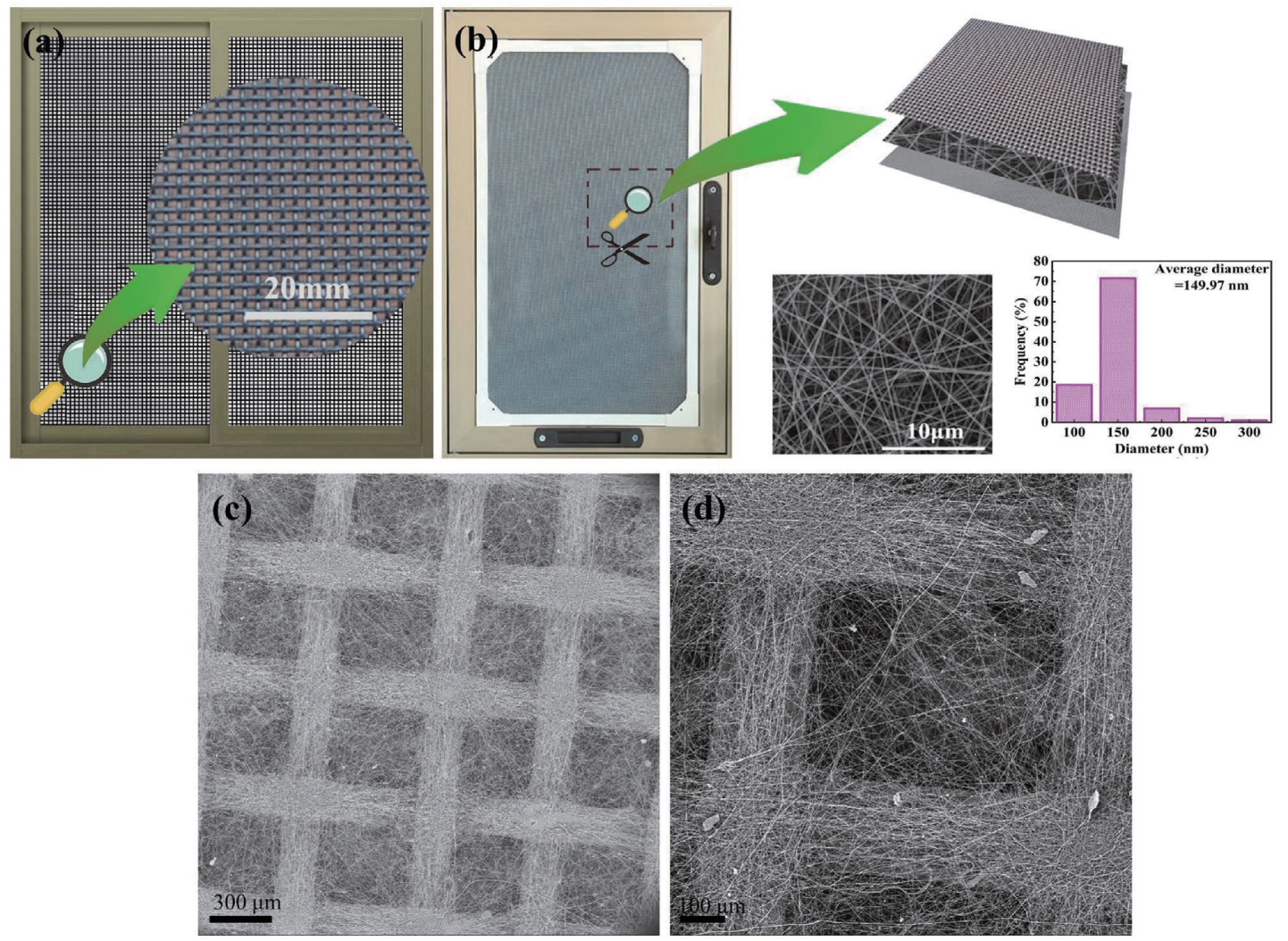

Fig. 4 (a) Picture of common window screen, (b) Physical picture of Nano window screen structure; SEM images of PAN nanofiber membrane at different multiples (c) 200x, and (d) 500x

important process parameters affecting the diameter of the electrospun fibers. Therefore, the diameter of the electrospun nanofibers is typically adjusted by the addition of an electrolyte [16]. In this study, $\mathrm{LiCl}$ was chosen for the adjustment of the conductivity of the spinning solution, and the effect of the fiber morphology was further investigated. As shown in Fig. 5, with the addition of $\mathrm{LiCl}$, the content of the finer fibers increased, the diameter distribution was concentrated at around 150-200 nm, and the average diameter decreased significantly. At the $\mathrm{LiCl}$ content of $0.02 \mathrm{wt} \%$, the diameter distribution of nanofibers was the narrowest, and the concentration was approximately $150 \mathrm{~nm}$, as shown in Fig. 5(c). However, as the amount of $\mathrm{LiCl}$ was gradually increased, the fiber diameter and dispersion increased, and there was a bonding phenomenon between adjacent fibers, forming a rod-like structure, as shown in Fig. 5(d). However, with the excessive addition of $\mathrm{LiCl}$, the higher salt content increased the viscosity of the solution, resulting in incomplete fiber splitting and twinning, which increased the dispersion of the fiber diameter. Therefore, in this study, the optimum $\mathrm{LiCl}$ content was determined to be $0.02 \mathrm{wt} \%$.

\subsection{Effect of receiving net speed on the filtration efficiency of the nanofiber}

To effectively block fine particles, the threedimensional gap between fibers needs to be sufficiently small, while the bulk density of the fibers in the non-woven membrane directly affects the pore size. In this study, the bulk density of the nanofibers was adjusted by the running speed of the electrospinning receiving mesh, and its effect on the filtration efficiency of the fiber membrane was analyzed [17]. As can be seen in Fig. 6, the filtration resistance decreased gradually with the increase in feeding speed, and their relationship was nearly linear. However, the change in the filtration efficiency was completely different. At a running speed less than $250 \mathrm{~mm} / \mathrm{min}$, the filtration efficiency of the nanofiber membrane was maintained above $90 \%$. When the running speed was greater than $250 \mathrm{~mm} / \mathrm{min}$, the filtration efficiency decreased rapidly. The bulk density of the fiber membrane at different feeding speeds could clearly be observed by SEM. At a running speed of $150 \mathrm{~mm} / \mathrm{min}$, the nanofibers were densely packed and the pore sizes of the fiber membrane were in the range of $1-5 \mu \mathrm{m}$, as shown in Fig. 7(a). Thus, the filtration efficiency was maintained at approximately $94 \%$. At a feeding speed of $350 \mathrm{~mm} /$ 

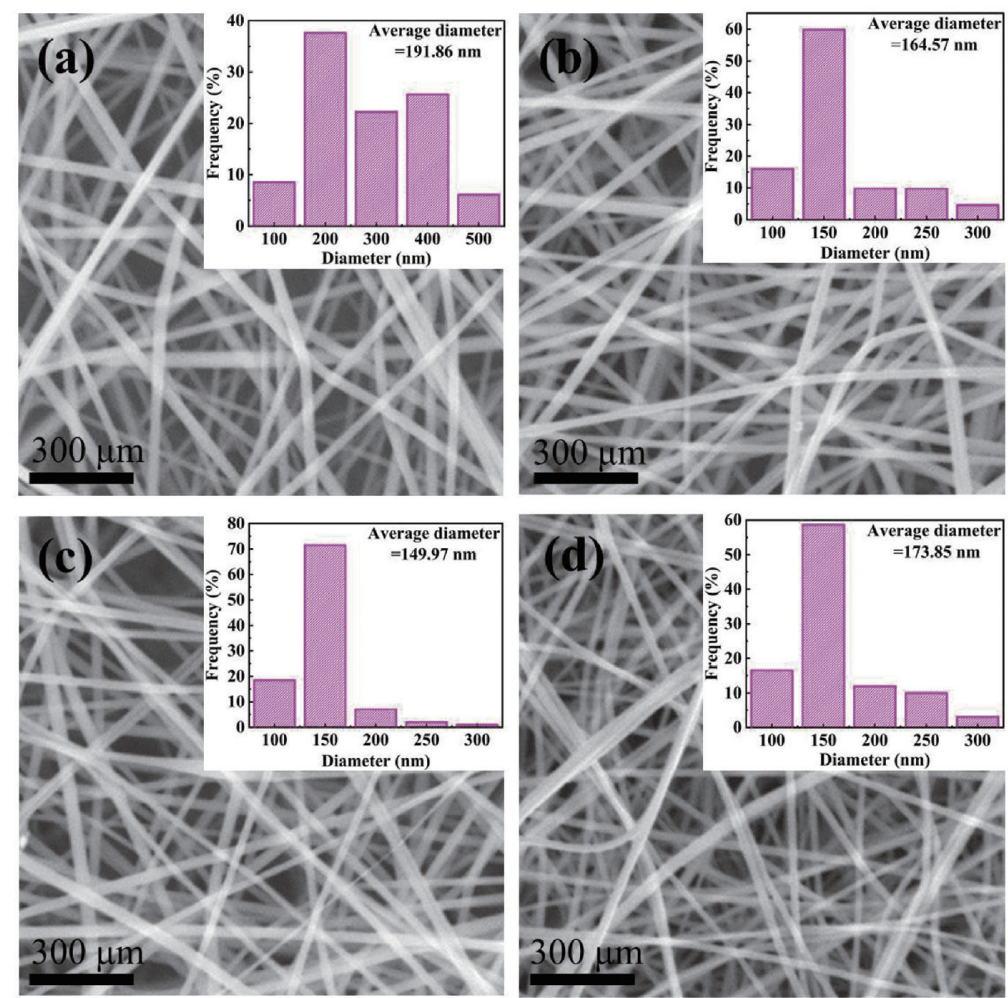

Fig. 5 SEM images and diameter distribution diagrams of different $\mathrm{LiCl}$ contents: (a) $0 \mathrm{wt} \%$, (b) $0.01 \mathrm{wt} \%$, (c) $0.02 \mathrm{wt} \%$, and (d) $0.03 \mathrm{wt} \%$

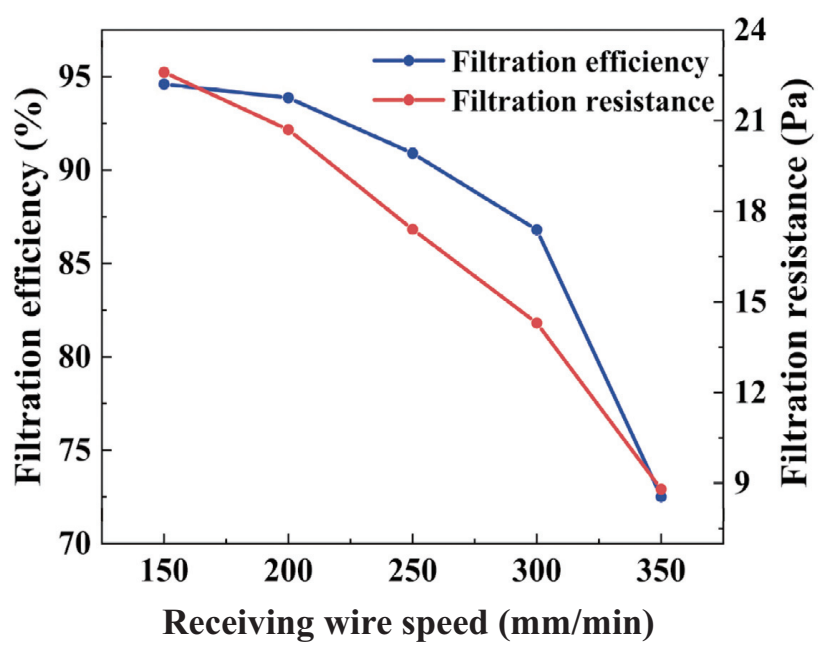

Fig. 6 Effect of receiving wire speed on the filtration performance of the PAN nanofiber membrane min, the pore size between the nanofibers increased to nearly $40 \mu \mathrm{m}$, and the filtration efficiency was decreased to approximately $72 \%$. Considering realistic demand and production efficiency, the running speed of the curtain was set to $250 \mathrm{~mm} / \mathrm{min}$.

\subsection{Effect of ultrasonic composite parameters on the performance of the window screen}

The ultrasonic compound process involves the changing of the mechanical energy of the highfrequency vibration into heat energy [18, 19], which results in the thermal adhesion of the thermoplastic polymer. Owing to its advantages including high efficiency, pollution-free operation, and simple equipment, it is one of the most widespread composite methods in the field of non-woven industry [20]. The
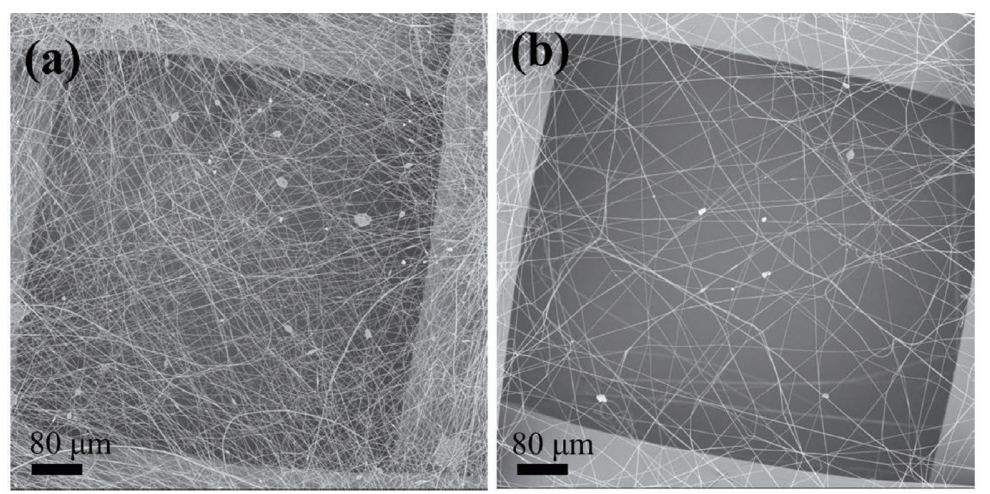

Fig. 7 SEM image of the PAN nanofiber membrane with a receiving network line speed of (a) $150 \mathrm{~mm} / \mathrm{min}$ and (b) $350 \mathrm{~mm} / \mathrm{min}$ 
anti-haze window screen designed in this study consists of three layers: a glass fiber mesh, a nanofiber membrane, and a polyester fine mesh. First, ultrasonic composite technology was used to bond window screens, and we studied the effect of ultrasonic composite parameters on its performance to assess the applicability of ultrasonic composites in this study. As shown in Fig. 8(a), the peel strength of the composite window screen gradually increased with the increase in the composite pressure. When the composite pressure increased to $2.6 \mathrm{MPa}$, the peel strength reached $211.04 \mathrm{~N} / \mathrm{m}$ that satisfied the requirements of practical applications. However, under this combined pressure, the filtration efficiency of the window screen decreased sharply. The SEM morphology showed that in this case, the front and back screens in the bonding point area were damaged, and the nanofiber membrane also had holes; thus, the filtration efficiency decreased rapidly. This suggests that the ultrasonic composite energy was too high to bond the nanofiber membrane materials.

\subsection{Effect of hot melt adhesive composite parameters on the performance of the window screen}

As discussed in the previous section, the ultrasonic process was not suitable for the adhesion of nanofiber membranes. Therefore, hot melt adhesive composite technology was employed to complete the bonding of the window screen. An environmentally friendly and high-performance adhesive resin, PUR [21-23], was chosen for this purpose. We studied the effect of the PUR amount on the performance of the composite window screen. The results showed that the peel strength increased with the increase of the coating amount. When the coating amount of PUR reached $1.2 \mathrm{~g} / \mathrm{m}^{2}$, the peel strength was $307.46 \mathrm{~N} / \mathrm{m}$. As the coating amount continued to increase, the peel strength remained nearly unchanged (Fig. 10(a)). The filter resistance of the composite window screen increased with the increase in the coating amount, but the filter efficiency remained constant. As the coating surface was a net structure, when all grid lines were coated with PUR, the bonding area saturated and the strength of the glass also became maximal. Therefore, the increase in the coating amount had little effect on the adhesion, as shown in Fig. 11(b). However, if the coating amount was too high, the glue flowed over the screen, resulting in a constant increase in the filter resistance, as shown in Fig. 11(d). Nevertheless, the (a)

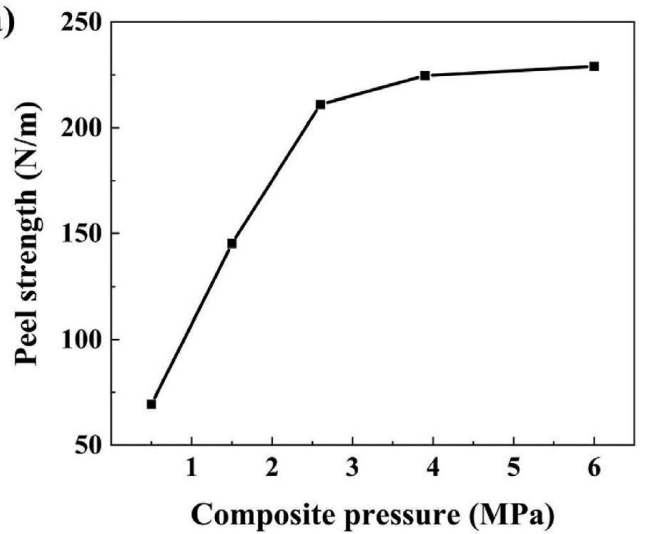

(b)

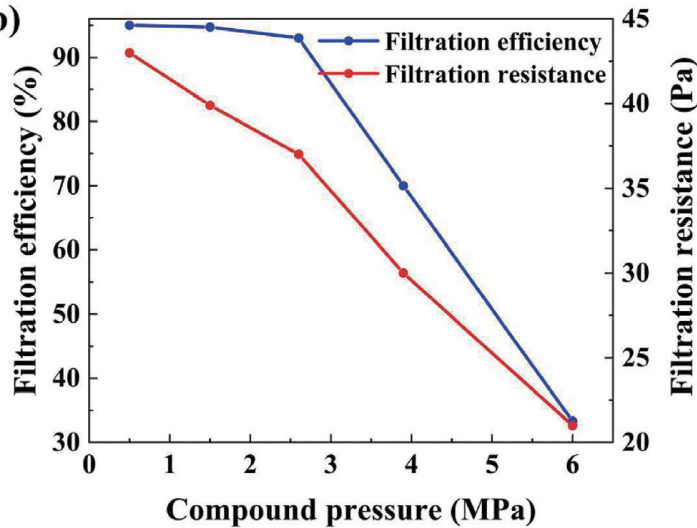

Fig. 8 Effect of compound pressure on the performance of anti-haze and haze screens: (a) peel strength and (b) filter performance
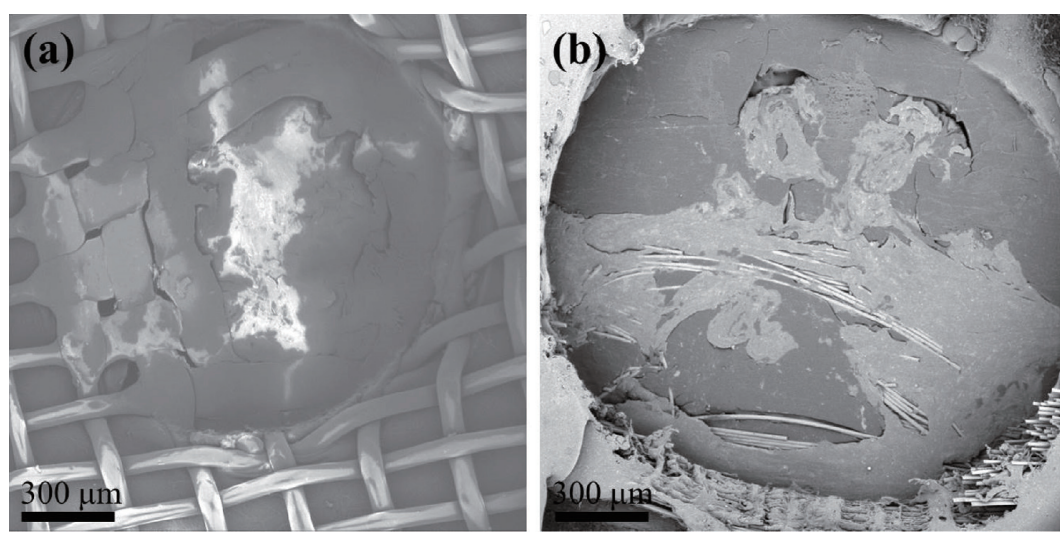

Fig. 9 SEM image of the ultrasonic composite anti-haze screen: (a) front and (b) back side 

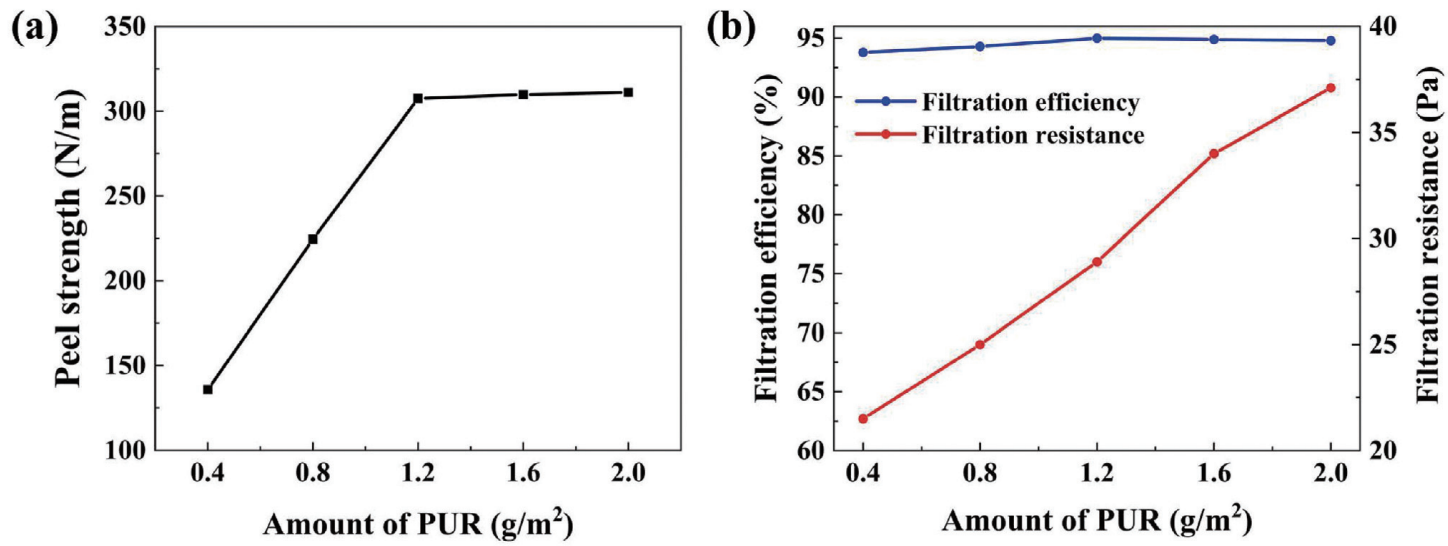

Fig. 10 Effect of the amount of PUR glue on the performance of anti-haze screens: (a) peel strength and (b) filtration performance
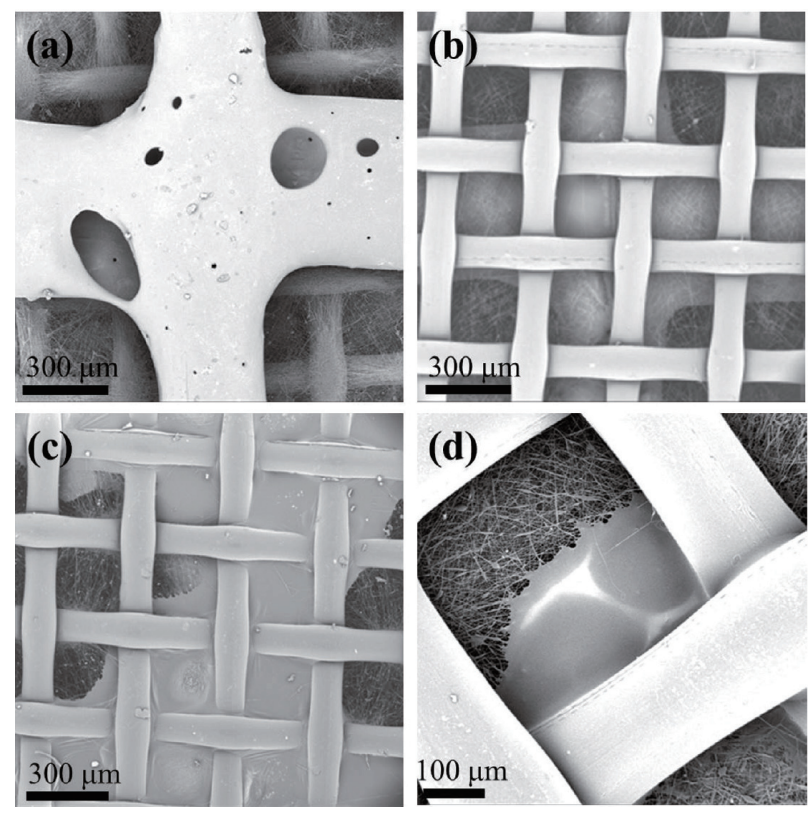

Fig. 11 SEM image of hot melt adhesive composite anti-haze screen

entire bonding process did not damage the nanofiber membrane; thus, it had little effect on the filtration efficiency.

During the bonding process of the PUR adhesive, the $-\mathrm{NCO}$ group reacts with $\mathrm{H}_{2} \mathrm{O}$ in the air to form a cross-linking structure; which is a very slow process [24,25]. Therefore, after the multi-layer mesh is coated with PUR and the layers are stacked together, they need to be held for a certain time to bond and solidify. In this study, the change in the peel strength of the composite window screen was analyzed as a function of storage time. As can be seen in Fig. 12, the peel strength of the window screen increased rapidly in $24 \mathrm{~h}$ with the increase in the storage time, the growth rate decreased sharply after $24 \mathrm{~h}$, and after $36 \mathrm{~h}$ the strength was constant. Considering this, the optimal storage time of the

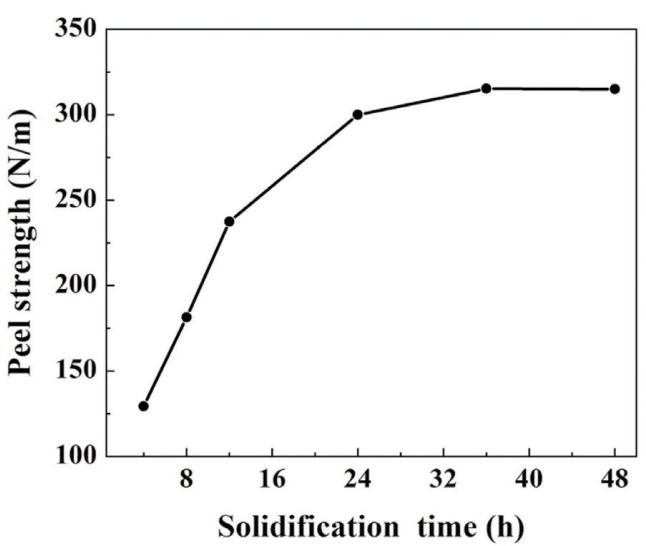

Fig. 12 Relationship between curing time and peel strength

window screen after the hot melt adhesive compound was set to $24 \mathrm{~h}$.

\section{Conclusions}

In this study, a sandwich structure of a nanofiber anti-haze window screen was designed, which was composed of a 120 mesh polyester screen, a nanofiber membrane as the middle layer, and a 30 mesh glass fiber screen as the protective layer. The analysis of the spinning process showed that at a $\mathrm{LiCl}$ content of $0.02 \mathrm{wt} \%$, the average diameter of the nanofibers was the smallest, and the distribution was higher.

In this study, we analyzed the effect of ultrasonic composite technology and hot adhesive compounding technology on the performance of an anti-haze window screen. The results show that glass fiber mesh, nanofiber membrane, and polyester fiber mesh can be bonded together by ultrasonic compounding. However, ultrasonic energy can destroy the nanofiber membrane at the bonding point, which decreases the efficiency of the window screen. In the hot adhesive 
compounding process, with an increase in the coating amount, the peel strength of the window screen made of hot melt adhesive was improved, and the filtration efficiency was nearly constant and maintained at approximately 95\%, but the filtration resistance increased. In this study, the optimal amount of glue was $1.2 \mathrm{~g} / \mathrm{m}^{2}$ and the bonding time was $24 \mathrm{~h}$.

It can be seen that hot melt adhesive composite technology is a suitable composite process for fabricating nanofiber window screens, with simple technology and controllable parameters, which are beneficial to the industrial manufacturing of anti-haze window screens.

\section{Acknowledgments}

This work is supported by National Natural Science Foundation of China (22002193, 51803244 and U2004178), Key Scientific Research Projects of Higher Education Institutions in Henan Province (20A540002 and 21A540005), and Youth talent support project of Henan Province of China (2020 HYTP 032). We would like to thank Editage (www.editage.cn) for English language editing.

\section{References}

1. B. Michael, C. Barbara and A. Robert, J. Am. Coll. Cardiol., 77, 1684 (2021).

2. A. Almetwally, M. Bin-Jumah and A. Ahmed, Environ. Sci. Pollut. R., 27, 24815 (2020).

3. G. S. Al-Kindi, D. R. Brook and S. Biswal, Nat. Rev. Cardiol., 17, 656 (2020).

4. A. Tomczak A, B. A. Miller and A. S. Weichenthal, Int. J. Cancer, 139, 1958 (2016).

5. X. Li, L. Jin and H. Kan, Nature, 570, 437 (2019).
6. R. Zhang, C. Liu and G. Zhou, Nano Res., 11, 3182 (2018).

7. A. R. Silva, J. J. West and Y. Zhang, Environ. Res. Lett., 8, 5 (2013).

8. E. J. Fisher, S. Loft and S. C. Ulrik, Am. J. Resp. Crit. Care., 194, 855 (2016).

9. X. Zhao, Y. Li and T. Hua, Small, 13, 1603306 (2017).

10. F. W. W. Leung, Y. W 0. Y. Hau and F. H. Choy, Sep. Purif. Technol., 206, 26 (2018).

11. H. Liu, C. Cao and J. Huang, Nanoscale, 12, 437 (2020).

12. A. Cherpinski, S. Torres-Giner and L. Cabedo, Food. Addit. Contam. A, 34, 1817 (2017).

13. M. Zhu, J. Han and F. Wang, Macromol. Mater. Eng., 302, 235 (2017).

14. H. Zhang, Y. Xie and Y. Song, Colloid. Surface. A, 126831 (2021).

15. SAC/TC 209, Standards Press of China, 2009.

16. R. S. Ardkapan, S. M. Johnson and S. Yazdi, J. Aerosol. Sci., 72, 14 (2014).

17. Y. Yang, S. Zhang and X. Zhao, Sep. Purif. Technol., 152, 14 (2015).

18. W. Eileen, Nonwovens Industry, 9, 44 (2003).

19. S. S. Volkov, Mashinostroeni, 21 (2005).

20. S. Sherrit andA. S. Askins, SPIE, 4701, 353 (2002).

21. A. Mukhopadhyay and K. V. Midha, J. Ind. Text., 38, 17 (2008).

22. Y. Zhou, W. Xue and Z. Zeng, J. Adhes. Sci. Technol., 27, 670 (2015).

23. W. Pall, Pigment \& Resine Technology, 26, 300 (1997).

24. A. Mukhopadhyay and K. V. Midha, J Ind. Text., 37, 225 (2008).

25. B. Khalid, X. Bai and H. Wei, Nano Lett., 17, 1140 (2017). 